\title{
Asymptotic Stability of Sampled-data Piecewise Affine Slab Systems *
}

\author{
Miad Moarref, Luis Rodrigues \\ Department of Electrical and Computer Engineering, Concordia University, Montreal, QC, H3G 2W1, Canada
}

\begin{abstract}
This paper addresses stability analysis of closed-loop sampled-data piecewise affine (PWA) slab systems. In particular, we study the case in which a PWA plant is in feedback with a discrete-time emulation of a PWA controller. We consider the sampled-data system as a continuous-time system with a variable time delay. The contributions of this work are threefold. First, we present a modified LyapunovKrasovskii functional (LKF) for studying PWA systems with time delays that is less conservative when compared to previously suggested alternatives. Second, based on the new LKF, sufficient conditions are provided for asymptotic stability of sampled-data PWA slab systems to the origin. These conditions become Linear Matrix Inequalities (LMIs) in the case of a piecewise linear (PWL) controller. Finally, we present an algorithm for finding a lower bound on the maximum delay that preserves asymptotic stability. Therefore, the output of the algorithm provides an upper bound on the minimum sampling frequency that guarantees asymptotic stability of the sampled data system. The new results are successfully applied to a unicycle example.
\end{abstract}

Key words: Piecewise affine systems; Sampled-data systems; Time-delay systems; Lyapunov stability.

\section{Introduction}

PWA systems form a special class of hybrid systems that is often considered as a framework for modeling and approximating nonlinearities that arise in physical systems; Rodrigues and How (2001). Stability analysis of continuoustime PWA systems was addressed using Lyapunov-based methods in Johansson and Rantzer (1998); Hassibi and Boyd (1998); Johansson (2002); Rodrigues (2004). Lyapunovbased synthesis methods for PWA systems were presented in Hassibi and Boyd (1998); Johansson (2002); Rodrigues and Boyd (2005); Samadi and Rodrigues (2009a). However, to be implementable in microprocessors, the resulting continuous-time controllers must be emulated as a discretetime controller. For a general framework for the design of nonlinear controllers using the emulation method, the reader is referred to Laila et al. (2002). For a direct discrete-time controller design based on an approximate discrete-time model of the plant, we refer the reader to Nešić et al. (1999); Nešić and Teel (2004).

While sampled-data control of linear systems is a wellstudied subject (e.g. see Chen and Francis (1995)), its extension to PWA systems has not received many research contri-

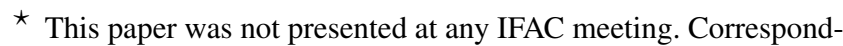
ing author L. Rodrigues. Tel. +1 514 8482424x3135; Fax +1 514 8482802.
}

Email addresses: m_moareencs . concordia.ca (Miad Moarref), luisrodeencs.concordia.ca (Luis Rodrigues). butions. The term "sampled-data PWA system" was probably used for the first time in Azuma and Imura (2003); Imura (2003), although the systems described there do not possess the typical structure of a continuous-time plant being controlled by a discrete-time controller. By contrast, Rodrigues (2007) addresses the classical structure of a sampled-data system in which a continuous-time system is controlled in discrete-time inside a computer. Assuming constant sampling rate, the author studies the stability of sampled-data PWA systems using a quadratic Lyapunov function. The paper provides a set of LMIs as sufficient conditions for exponential convergence of the sampled-data system to an invariant set containing the origin.

In sampled-data systems, the discrete-time controller can also be modeled as a continuous-time controller with time varying delay. The time-delay representation has been implemented in nonlinear and linear sampled-data systems using Razumikhin-type theorems (Teel et al. (1998)), and LKFs (Naghshtabrizi et al. (2008)). Following the time-delay approach, Samadi and Rodrigues (2009b) study the stability of sampled-data PWA systems with variable sampling rate. The paper uses an LKF to prove that if a set of LMIs are satisfied, the trajectories of the sampled-data system converge to an invariant set containing the origin.

In contrast to previous work, we address asymptotic stability to the origin rather than stability to an invariant set for sampled-data PWA slab systems. To the best of our knowledge, asymptotic stability of sampled-data PWA systems to 
the origin was not proved before. We study a continuoustime PWA slab plant in feedback with a PWA slab controller that appears between a sampler, with variable sampling rate, and a zero-order-hold. The contributions of this work are threefold. First, we present a modified LKF for studying PWA systems with time delays that is less conservative when compared to previously suggested alternatives; Samadi and Rodrigues (2009b). Second, based on the new LKF, sufficient conditions are provided for asymptotic stability of sampled-data PWA slab systems to the origin. Finally, following the time-delay approach, we present an algorithm for finding a lower bound on the maximum delay that preserves asymptotic stability. This result provides an upper bound on the minimum sampling frequency that guarantees asymptotic stability of the sampled data system. Preliminary results on a simpler version of this problem addressing the case of PWL controllers can be found in Moarref and Rodrigues (2011). That case will only be presented in this paper as a corollary.

The paper is organized as follows. Section 2 presents basic information about sampled-data PWA slab systems. In Section 3, a modified LKF is introduced first. Next, we present a theorem that provides sufficient conditions for asymptotic stability of sampled-data PWA slab systems. Furthermore, we present an algorithm for finding a lower bound on the maximum delay that preserves asymptotic stability. Finally, the new results are applied to a unicycle example in Section 4 , followed by conclusions.

\section{Preliminaries}

Consider the PWA slab system

$$
\dot{x}(t)=A_{i} x(t)+a_{i}+B u(t), \text { for } x(t) \in \mathcal{R}_{i} \text { and } i \in \mathcal{I},
$$

where $x \in \mathcal{X} \subset \mathbb{R}^{n_{x}}$ denotes the state vector, $A_{i} \in \mathbb{R}^{n_{x} \times n_{x}}$, $a_{i} \in \mathbb{R}^{n_{x}}, B \in \mathbb{R}^{n_{x} \times n_{u}}, u \in \mathbb{R}^{n_{u}}$ is the control input, and $\mathcal{I}=\{1, \ldots, M\}$ is the set of indices of the slab regions $\mathcal{R}_{i}$ that partition the state space $\mathcal{X}$. The slab regions are defined as

$$
\mathcal{R}_{i}=\left\{x \in \mathbb{R}^{n_{x}} \mid \sigma_{i}<c^{T} x<\sigma_{i+1}\right\}, i \in \mathcal{I},
$$

where $c \in \mathbb{R}^{n_{x}}, c \neq 0$, and $\sigma_{1}<\sigma_{2}<\ldots<\sigma_{M+1}$ are finite scalars. We denote the closure of $\mathcal{R}_{i}$ by $\overline{\mathcal{R}_{i}}$. The state space is represented by the union of the closure of all regions, i.e.

$$
\mathcal{X}=\bigcup_{i \in \mathcal{I}} \overline{\mathcal{R}_{i}}=\left\{x \in \mathbb{R}^{n_{x}} \mid \sigma_{1} \leq c^{T} x \leq \sigma_{M+1}\right\}
$$

Based on (3) and (2), the state space $\mathcal{X}$ and the regions $\mathcal{R}_{i}$ are only bounded in the direction of vector $c$. Each slab region $\mathcal{R}_{i}$ can also be described by a degenerate ellipsoid as

$$
\mathcal{R}_{i}=\left\{x||\left|L_{i} x+l_{i}\right| \mid<1\right\},
$$

where $L_{i}=2 c^{T} /\left(\sigma_{i+1}-\sigma_{i}\right)$ and $l_{i}=-\left(\sigma_{i+1}+\sigma_{i}\right) /\left(\sigma_{i+1}-\right.$ $\left.\sigma_{i}\right)$; Rodrigues and Boyd (2005).
Lemma 1 For the slab regions defined in (4), $x \in \mathcal{R}_{i}$ if and only if

$$
\left[\begin{array}{ll}
x^{T} & 1
\end{array}\right]\left[\begin{array}{cc}
L_{i}^{T} L_{i} & L_{i}^{T} l_{i} \\
l_{i} L_{i} & l_{i}^{2}-1
\end{array}\right]\left[\begin{array}{l}
x \\
1
\end{array}\right]<0
$$

PROOF. According to (4), $x \in \mathcal{R}_{i}$ if and only if ||$L_{i} x+l_{i} \|<1$. Therefore,

$$
\begin{aligned}
x \in \mathcal{R}_{i} & \Longleftrightarrow\left(L_{i} x+l_{i}\right)^{2}<1 \\
& \Longleftrightarrow\left(\left[\begin{array}{ll}
x^{T} & 1
\end{array}\right]\left[\begin{array}{c}
L_{i}^{T} \\
l_{i}
\end{array}\right]\right)\left(\left[\begin{array}{ll}
L_{i} & l_{i}
\end{array}\right]\left[\begin{array}{l}
x \\
1
\end{array}\right]\right)<1 \\
& \Longleftrightarrow\left[\begin{array}{ll}
x^{T} & 1
\end{array}\right]\left[\begin{array}{cc}
L_{i}^{T} L_{i} & L_{i}^{T} l_{i} \\
l_{i} L_{i} & l_{i}^{2}-1
\end{array}\right]\left[\begin{array}{l}
x \\
1
\end{array}\right]<0 .
\end{aligned}
$$

Let a PWA controller for (1) be defined by

$$
u(t)=K_{i} x(t)+k_{i}, \text { for } x(t) \in \mathcal{R}_{i},
$$

where $K_{i} \in \mathbb{R}^{n_{u} \times n_{x}}$ and $k_{i} \in \mathbb{R}^{n_{u}}$. We now present the assumptions used in this work.

Assumption 1 The vector field of the open-loop system (1) for $u(t)=0$ is continuous across the boundaries of any neighboring regions.

We denote the region containing the origin by $\mathcal{R}^{*}$.

Assumption 2 The open-loop and closed-loop systems are linear in $\mathcal{R}^{*}$, i.e. $a_{i}=0$ and $k_{i}=0$ for $\mathcal{R}_{i}=\mathcal{R}^{*}$.

Assumption 3 The state vector is measured at the sampling instants $t_{n}, n \in \mathbb{N}$, where $0<t_{\epsilon} \leq t_{n+1}-t_{n} \leq \tau$ for all $n \in \mathbb{N}$.

The positive constant $t_{\epsilon}$ is an arbitrary small number that models the fact that two transmissions cannot occur simultaneously in practice. It is also used later to rule out the existence of the Zeno phenomenon. The number $\tau$ denotes the longest interval between two consecutive sampling times.

According to Assumption 3, the control input is rewritten as $u(t)=K_{j} x_{t_{n}}+k_{j}$, for $t \in\left[t_{n}, t_{n+1}\right), x_{t_{n}} \in \mathcal{R}_{j}$, and $j \in \mathcal{I}$ where $x_{t_{n}}=x\left(t_{n}\right)$. We denote the time elapsed since the last sampling instant by

$$
\rho(t)=t-t_{n}, \text { for } t \in\left[t_{n}, t_{n+1}\right) .
$$


Assuming $x(t) \in \mathcal{R}_{i}$ and $x_{t_{n}} \in \mathcal{R}_{j}$, for $t \in\left[t_{n}, t_{n+1}\right)$, we can rewrite (1) as

$$
\begin{aligned}
\dot{x}(t) & =A_{i} x(t)+a_{i}+B\left(K_{j} x_{t_{n}}+k_{j}\right) \\
& =A_{i} x(t)+a_{i}+B\left(K_{i} x_{t_{n}}+k_{i}\right)+B w(t),
\end{aligned}
$$

where $w \in \mathbb{R}^{n_{u}}$ is a piecewise constant vector defined by

$$
w(t)=\left(K_{j}-K_{i}\right) x_{t_{n}}+\left(k_{j}-k_{i}\right)
$$

The vector $w$ is associated with the fact that the state and its most recent sample can possibly be in different regions.

To be of later use in the proofs we must define bounds on the mismatch between controller gain matrices $K_{i}$ and affine vectors $k_{i}, i \in \mathcal{I}$. To that end, let $B_{\mu}(0)$ be the ball with radius $\mu>0$ centered at the origin and $\mathcal{I}_{\mu}=\left\{p \in \mathcal{I} \mid \overline{\mathcal{R}_{p}} \bigcap B_{\mu}(0) \neq \emptyset\right\}$. We define non-negative scalars $\Delta K_{\mu}$ and $\delta k_{\mu}$ as

$$
\Delta K_{\mu}=\max _{i \in \mathcal{I}, j \in \mathcal{I}_{\mu}}\left\|K_{j}-K_{i}\right\|, \delta k_{\mu}=\max _{i \in \mathcal{I}, j \in \mathcal{I}_{\mu}}\left\|k_{j}-k_{i}\right\| .
$$

Furthermore, let non-negative scalars $\Delta K$ and $\delta k$ be defined as

$$
\Delta K=\max _{i, j \in \mathcal{I}}\left\|K_{j}-K_{i}\right\|, \quad \delta k=\max _{i, j \in \mathcal{I}}\left\|k_{j}-k_{i}\right\| .
$$

We denote the zero matrix and the identity matrix of the appropriate size by 0 and $I$, respectively. The symmetric entries of a symmetric matrix are represented by $\star$. A block diagonal matrix with diagonal entries $d_{1}, \ldots, d_{m}$ is denoted by $\operatorname{diag}\left(d_{1}, \ldots, d_{m}\right)$. The following lemma presents a bound on the vector $w$ which is used in the proof of the main result.

Lemma 2 For $t \in\left[t_{n}, t_{n+1}\right)$, if $\left\|x_{t_{n}}\right\|<\mu$, then

$$
\left[\begin{array}{cc}
-I & \left(\Delta K_{\mu} \mu+\delta k_{\mu}\right) \boldsymbol{I} \\
I & \left(\Delta K_{\mu} \mu+\delta k_{\mu}\right) \boldsymbol{I}
\end{array}\right]\left[\begin{array}{c}
w(t) \\
1
\end{array}\right] \succ 0
$$

where $\mathbf{I}^{T}=[1 \cdots 1]_{1 \times n_{u}}$ and $\succ$ represents an elementwise inequality.

PROOF. If $\left\|x_{t_{n}}\right\|<\mu$, according to (7) and (8) we can write

$$
\begin{aligned}
\|w(t)\| & \leq\left\|K_{j}-K_{i}\right\|\left\|x_{t_{n}}\right\|+\left\|k_{j}-k_{i}\right\| \\
& <\Delta K_{\mu} \mu+\delta k_{\mu} .
\end{aligned}
$$

For single input systems, inequality (11) can be written as

$$
\left[\begin{array}{cc}
-1 & \Delta K_{\mu} \mu+\delta k_{\mu} \\
1 & \Delta K_{\mu} \mu+\delta k_{\mu}
\end{array}\right]\left[\begin{array}{c}
w(t) \\
1
\end{array}\right] \succ 0 .
$$

For the case of multi-input systems, a more conservative inequality can be written as (10), i.e. the absolute value of each element of $w$ is less than $\Delta K_{\mu} \mu+\delta k_{\mu}$.

\section{Main Results}

In this section, we first present a modified LKF for studying PWA systems with time delays. Let

$$
V: \mathcal{X} \times \mathcal{C}([-\tau, 0], \mathcal{X}) \times[0, \tau] \rightarrow \mathbb{R}^{+}
$$

be an LKF, where $\mathcal{C}([-\tau, 0], \mathcal{X})$ is the space of absolutely continuous functions mapping the interval $[-\tau, 0]$ to $\mathcal{X}$. We define $x_{t} \in \mathcal{C}$ as $x_{t}(r)=x(t+r),-\tau \leq r \leq 0$, and denote its norm by

$$
\left\|x_{t}\right\|_{\mathcal{C}}=\sup _{-\tau \leq r \leq 0}\left\|x_{t}(r)\right\|
$$

(see Hale and Lunel (1993), Section 2.1). We now define

$$
V\left(x, x_{t}, \rho\right)=V_{1}(x)+V_{2}\left(x_{t}\right)+V_{3}\left(x_{t}, \rho\right),
$$

with

$$
\begin{aligned}
V_{1}(x) & =x^{T}(t) P x(t), \\
V_{2}\left(x_{t}\right) & =\int_{-\tau}^{0} \int_{t+r}^{t}\left[\dot{x}(s)-B\left(K_{j} x_{t_{n}}+k_{j}\right)\right]^{T} \\
R\left[\dot{x}(s)-B\left(K_{j} x_{t_{n}}+k_{j}\right)\right] \mathrm{d} s \mathrm{~d} r, & \\
V_{3}\left(x_{t}, \rho\right) & =(\tau-\rho)\left(x(t)-x_{t_{n}}\right)^{T} X\left(x(t)-x_{t_{n}}\right),
\end{aligned}
$$

where $P, R$, and $X$ are symmetric positive definite matrices in $\mathbb{R}^{n_{x} \times n_{x}}, t_{n} \leq t$ is the most recent sampling instant, and $j$ is the index of the region containing the most recent sampled state, i.e. $x_{t_{n}} \in \mathcal{R}_{j}$.

Note that the second component of the LKF introduced in (12) is different from its corresponding term in previously studied LKFs such as Naghshtabrizi et al. (2008); Samadi and Rodrigues (2009b). By subtracting $B\left(K_{j} x_{t_{n}}+k_{j}\right)$ from $\dot{x}$ in the definition of $V_{2}$, we omit an unfavorable positive definite term involving $w^{T} w$ from $\dot{V}$. This modification considerably improves the stability results as shown in Section 4. We now define stability in the context of retarded functional differential equations.

Definition [Gu et al. (2003)] The solution of (6a) is said to be uniformly stable if for any $\epsilon>0$, there is a $\delta=\delta(\epsilon)$ such that $\left\|x_{t_{0}}\right\|_{\mathcal{C}}<\delta$ implies $\|x(t)\|<\epsilon$ for $t \geq t_{0}$. The solution of (6a) is uniformly asymptotically stable if it is uniformly stable and there is $\delta_{a}>0$ such that for any $\eta>0$, there is a $T=T\left(\delta_{a}, \eta\right)$, such that $\left\|x_{t_{0}}\right\|_{\mathcal{C}}<\delta_{a}$ implies $\|x(t)\|<\eta$ for $t \geq t_{0}+T$.

The following theorem provides a set of sufficient conditions for which the trajectories of a sampled-data PWA slab system asymptotically converge to the origin. 
Theorem 1 Consider the sampled-data PWA slab system defined in (6b) and (7) subject to Assumptions 1-3. The system is uniformly asymptotically stable to the origin if there exist symmetric positive definite matrices $P, R$, and $X$, symmetric matrices $\Lambda_{i}$ with non-negative entries, matrices $\widetilde{N}$ and $N_{i}$, with appropriate dimensions, and positive scalars $\gamma, \theta<1, \eta, \lambda_{i}, \sigma$, and $\epsilon$, with $i \in \mathcal{I}$, satisfying

$$
\Delta K^{2} \gamma<\theta
$$

- for all $i$ such that $\mathcal{R}_{i} \neq \mathcal{R}^{*}$

$$
\Omega_{i}+\bar{\Omega}_{i}+\bar{\Omega}_{i}^{T}+\tau\left(\bar{M}_{1 i}+\bar{M}_{1 i}^{T}+\bar{M}_{2 i}\right)+S_{1 i}+D<0
$$

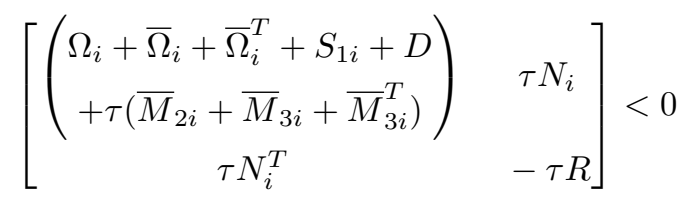

$$
\Omega_{i}+\bar{\Omega}_{i}+\bar{\Omega}_{i}^{T}+\tau\left(\bar{M}_{1 i}+\bar{M}_{1 i}^{T}+\bar{M}_{2 i}\right)
$$

$$
\left.\left[\begin{array}{cr}
\Omega_{i}+\bar{\Omega}_{i}+\bar{\Omega}_{i}^{T}+S_{1 i}+S_{3 i} \\
+\tau\left(\bar{M}_{2 i}+\bar{M}_{3 i}+\bar{M}_{3 i}^{T}\right)+\epsilon I
\end{array}\right) \quad \begin{array}{r}
\tau N_{i} \\
\tau N_{i}^{T}
\end{array}\right]<0 \quad-S_{1 i}+S_{3 i}+\epsilon I<0
$$

- for $i$ such that $\mathcal{R}_{i}=\mathcal{R}^{*}$

$$
\begin{aligned}
& \Omega_{i}+\tau\left(M_{1 i}+M_{1 i}^{T}+M_{2 i}\right)+S_{1 i}-S_{2 i}+D<0 \\
& {\left[\begin{array}{cc}
\Omega_{i}+\tau\left(M_{2 i}+M_{3 i}+M_{3 i}^{T}\right) \\
+S_{1 i}-S_{2 i}+D \\
\tau N_{i}^{T} & \tau N_{i} \\
\end{array}\right]<0}
\end{aligned}
$$

$\Omega_{i}+\tau\left(M_{1 i}+M_{1 i}^{T}+M_{2 i}\right)+S_{1 i}-S_{2 i}+S_{3 i}+\epsilon I<0$

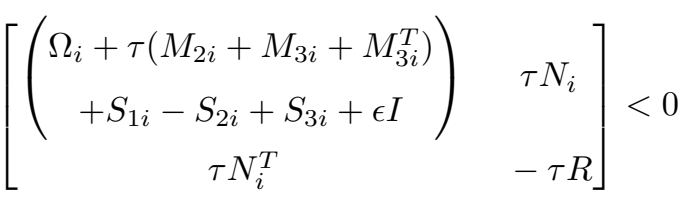

$$
\Psi_{i}+\tau \widetilde{M}_{1 i}+\widetilde{M}_{3 i}+\epsilon I<0
$$

$$
\left[\begin{array}{cc}
\Psi_{i}+\tau \widetilde{M}_{2 i}+\widetilde{M}_{3 i}+\epsilon I & \tau \widetilde{N}_{i} \\
\tau \widetilde{N}_{i}^{T} & -\tau R
\end{array}\right]<0
$$

where

$$
\Omega_{i}=\left[\begin{array}{cc}
\Psi_{i} & {\left[\begin{array}{cc}
P B & 0 \\
0 & 0
\end{array}\right]} \\
\star & 0
\end{array}\right]-\left[\begin{array}{llll}
N_{i} & -N_{i} & 0 & 0
\end{array}\right]^{T}
$$

$$
\begin{aligned}
& -\left[\begin{array}{llll}
N_{i} & -N_{i} & 0 & 0
\end{array}\right] \\
& \Psi_{i}=\left[\begin{array}{cc}
A_{i}^{T} P+P A_{i}-X & P B K_{i}+X \\
\star & -X
\end{array}\right] \text {, } \\
& \bar{\Omega}_{i}=\left[\begin{array}{llll}
P & 0 & 0 & 0
\end{array}\right]^{T}\left[\begin{array}{llll}
0 & 0 & 0 & a_{i}+B k_{i}
\end{array}\right] \text {, } \\
& \bar{M}_{1 i}=\left[\begin{array}{llll}
X & -X & 0 & 0
\end{array}\right]^{T}\left[\begin{array}{llll}
A_{i} & B K_{i} & B & a_{i}+B k_{i}
\end{array}\right] \text {, } \\
& \bar{M}_{2 i}=\left[\begin{array}{llll}
A_{i} & 0 & 0 & a_{i}
\end{array}\right]^{T} R\left[\begin{array}{llll}
A_{i} & 0 & 0 & a_{i}
\end{array}\right] \text {, } \\
& \bar{M}_{3 i}=N_{i}\left[\begin{array}{llll}
0 & B K_{i} & B & B k_{i}
\end{array}\right] \text {, } \\
& S_{1 i}=-\lambda_{i}\left(\left[\begin{array}{llll}
L_{i} & 0 & 0 & l_{i}
\end{array}\right]^{T}\left[\begin{array}{llll}
L_{i} & 0 & 0 & l_{i}
\end{array}\right]\right. \\
& \left.-\left[\begin{array}{llll}
0 & 0 & 0 & 1
\end{array}\right]^{T}\left[\begin{array}{llll}
0 & 0 & 0 & 1
\end{array}\right]\right) \\
& S_{2 i}=-\sigma\left(\left[\begin{array}{llll}
0 & L_{i} & 0 & l_{i}
\end{array}\right]^{T}\left[\begin{array}{llll}
0 & L_{i} & 0 & l_{i}
\end{array}\right]\right. \\
& \left.-\left[\begin{array}{llll}
0 & 0 & 0 & 1
\end{array}\right]^{T}\left[\begin{array}{llll}
0 & 0 & 0 & 1
\end{array}\right]\right) \\
& S_{3 i}=\left[\begin{array}{cccc}
0 & 0 & -I & \left(\Delta K_{\mu} \mu_{\tau}+\delta k_{\mu}\right) \boldsymbol{I} \\
0 & 0 & I & \left(\Delta K_{\mu} \mu_{\tau}+\delta k_{\mu}\right) \boldsymbol{l}
\end{array}\right]^{T} \Lambda_{i} \\
& {\left[\begin{array}{cccc}
0 & 0 & -I & \left(\Delta K_{\mu} \mu_{\tau}+\delta k_{\mu}\right) \boldsymbol{I} \\
0 & 0 & I & \left(\Delta K_{\mu} \mu_{\tau}+\delta k_{\mu}\right) \boldsymbol{l}
\end{array}\right]} \\
& \mu_{\tau}=\frac{\delta k}{\sqrt{\theta / \gamma}-\Delta K}, \\
& D=\operatorname{diag}(\eta I, I,-\gamma I, \eta), \\
& M_{1 i}=\left[\begin{array}{llll}
X & -X & 0 & 0
\end{array}\right]^{T}\left[\begin{array}{lllll}
A_{i} & B K_{i} & B & 0
\end{array}\right] \text {, } \\
& M_{2 i}=\left[\begin{array}{llll}
A_{i} & 0 & 0 & 0
\end{array}\right]^{T} R\left[\begin{array}{llll}
A_{i} & 0 & 0 & 0
\end{array}\right] \text {, } \\
& M_{3 i}=N_{i}\left[\begin{array}{llll}
0 & B K_{i} & B & 0
\end{array}\right] \text {, } \\
& \widetilde{M}_{1 i}=\left[\begin{array}{cc}
A_{i}^{T} X+X A_{i}+A_{i}^{T} R A_{i} & -A_{i}^{T} X+X B K_{i} \\
\star & -K_{i}^{T} B^{T} X-X B K_{i}
\end{array}\right] \text {, } \\
& \widetilde{M}_{2 i}=\left[\begin{array}{c}
0 \\
K_{i}^{T} B^{T}
\end{array}\right] \widetilde{N}_{i}^{T}+\widetilde{N}_{i}\left[\begin{array}{ll}
0 & B K_{i}
\end{array}\right]+\left[\begin{array}{cc}
A_{i}^{T} R A_{i} & 0 \\
\star & 0
\end{array}\right] \text {, } \\
& \widetilde{M}_{3 i}=-\left[\begin{array}{ll}
\widetilde{N}_{i} & -\widetilde{N}_{i}
\end{array}\right]^{T}-\left[\begin{array}{ll}
\widetilde{N}_{i} & -\widetilde{N}_{i}
\end{array}\right] \text {. }
\end{aligned}
$$

PROOF. Similar to the approach in Samadi and Rodrigues (2009b), it can be shown that the LKF (12) is positive definite, radially unbounded, and decrescent. The first two components, $V_{1}$ and $V_{2}$, are continuous functions. The last component, $V_{3}$, is equal to zero at the sampling instants $\left(\left.x(t)\right|_{t=t_{n}}=x_{t_{n}}\right)$ and greater than zero at other times. Therefore, the LKF is non-increasing at the sampling times. 
To prove uniform asymptotic stability of the closed-loop trajectories to the origin, it suffices to show that inequalities (13)-(23) are sufficient conditions for $V$ to be strictly decreasing between any two consecutive sampling times.

The time derivative of $V$ for $t \in\left(t_{n}, t_{n+1}\right)$ is computed as follows. First, the time derivative of $V_{1}$ is

$$
\dot{V}_{1}=\dot{x}^{T} P x+x^{T} P \dot{x} .
$$

Second, applying the Leibniz integral rule to $V_{2}$ yields

$$
\begin{gathered}
\dot{V}_{2}=\int_{-\tau}^{0}\left[\dot{x}-B\left(K_{j} x_{t_{n}}+k_{j}\right)\right]^{T} R \\
{\left[\dot{x}-B\left(K_{j} x_{t_{n}}+k_{j}\right)\right] \mathrm{d} r} \\
-\int_{-\tau}^{0}\left[\dot{x}(t+r)-B\left(K_{j} x_{t_{n}}+k_{j}\right)\right]^{T} R \\
{\left[\dot{x}(t+r)-B\left(K_{j} x_{t_{n}}+k_{j}\right)\right] \mathrm{d} r .}
\end{gathered}
$$

According to (5), we have $\rho<\tau$. Therefore,

$$
\begin{aligned}
& \dot{V}_{2} \leq \tau {\left[\dot{x}-B\left(K_{j} x_{t_{n}}+k_{j}\right)\right]^{T} R\left[\dot{x}-B\left(K_{j} x_{t_{n}}+k_{j}\right)\right] } \\
&- \int_{-\rho}^{0}\left[\dot{x}(t+r)-B\left(K_{j} x_{t_{n}}+k_{j}\right)\right]^{T} \\
&= R\left[\dot{x}(t+r)-B\left(K_{j} x_{t_{n}}+k_{j}\right)\right] \mathrm{d} r \\
&-\int_{t-\rho}^{t}\left[\dot{x}(v)-B\left(K_{j} x_{t_{n}}+k_{j}\right)\right]^{T} R\left[\dot{x}-B\left(K_{j} x_{t_{n}}+k_{j}\right)\right] \\
& R\left[\dot{x}(v)-B\left(K_{j} x_{t_{n}}+k_{j}\right)\right] \mathrm{d} v .
\end{aligned}
$$

Since $R$ is positive definite, for any arbitrary time varying vector $h_{i}(t) \in \mathbb{R}^{n_{x}}$ we can write

$$
\begin{aligned}
& {\left[\left(\dot{x}(v)-B\left(K_{j} x_{t_{n}}+k_{j}\right)\right)^{T} \quad h_{i}^{T}\right]} \\
& {\left[\begin{array}{cc}
R & -I \\
-I & R^{-1}
\end{array}\right]\left[\begin{array}{c}
\dot{x}(v)-B\left(K_{j} x_{t_{n}}+k_{j}\right) \\
h_{i}
\end{array}\right] \geq 0 .}
\end{aligned}
$$

Therefore,

$$
\begin{aligned}
& -\left[\dot{x}(v)-B\left(K_{j} x_{t_{n}}+k_{j}\right)\right]^{T} R\left[\dot{x}(v)-B\left(K_{j} x_{t_{n}}+k_{j}\right)\right] \\
& \leq h_{i}^{T} R^{-1} h_{i}-\left[\dot{x}(v)-B\left(K_{j} x_{t_{n}}+k_{j}\right)\right]^{T} h_{i} \\
& \quad-h_{i}^{T}\left[\dot{x}(v)-B\left(K_{j} x_{t_{n}}+k_{j}\right)\right] .
\end{aligned}
$$

Integrating both sides from $t-\rho$ to $t$, we have

$$
\begin{aligned}
& -\int_{t-\rho}^{t}\left[\dot{x}(v)-B\left(K_{j} x_{t_{n}}+k_{j}\right)\right]^{T} \\
& \quad R\left[\dot{x}(v)-B\left(K_{j} x_{t_{n}}+k_{j}\right)\right] \mathrm{d} v \\
& \quad \leq \rho h_{i}^{T} R^{-1} h_{i}-\left[x-x_{t_{n}}-\rho B\left(K_{j} x_{t_{n}}+k_{j}\right)\right]^{T} h_{i} \\
& \quad-h_{i}^{T}\left[x-x_{t_{n}}-\rho B\left(K_{j} x_{t_{n}}+k_{j}\right)\right] .
\end{aligned}
$$

Here, we used the facts that for $v \in[t-\rho, t], u=K_{j} x_{t_{n}}+k_{j}$ is constant and therefore $\dot{x}(v)$ is continuous by Assumption 1 , and $t-\rho=t_{n}$. Replacing (27) in (26), we have

$$
\begin{aligned}
\dot{V}_{2} \leq & \tau\left[\dot{x}-B\left(K_{j} x_{t_{n}}+k_{j}\right)\right]^{T} R\left[\dot{x}-B\left(K_{j} x_{t_{n}}+k_{j}\right)\right] \\
& +\rho h_{i}^{T} R^{-1} h_{i}-\left[x-x_{t_{n}}-\rho B\left(K_{j} x_{t_{n}}+k_{j}\right)\right]^{T} h_{i} \\
& -h_{i}^{T}\left[x-x_{t_{n}}-\rho B\left(K_{j} x_{t_{n}}+k_{j}\right)\right] .
\end{aligned}
$$

Using (7) to replace $K_{j} x_{t_{n}}+k_{j}$ by $\left(K_{i} x_{t_{n}}+k_{i}\right)+w$ in the last two components of (28) yields

$$
\begin{aligned}
\dot{V}_{2} \leq & {\left[\dot{x}-B\left(K_{j} x_{t_{n}}+k_{j}\right)\right]^{T} R\left[\dot{x}-B\left(K_{j} x_{t_{n}}+k_{j}\right)\right] } \\
& +\rho h_{i}^{T} R^{-1} h_{i} \\
& -\left[x-x_{t_{n}}-\rho B\left(\left(K_{i} x_{t_{n}}+k_{i}\right)+w\right)\right]^{T} h_{i} \\
& -h_{i}^{T}\left[x-x_{t_{n}}-\rho B\left(\left(K_{i} x_{t_{n}}+k_{i}\right)+w\right)\right]
\end{aligned}
$$

From (5) we have $\dot{\rho}=1$. Hence, the time derivative of $V_{3}$ is computed as

$$
\begin{aligned}
\dot{V}_{3}= & (\tau-\rho)\left[\dot{x}^{T} X\left(x-x_{t_{n}}\right)\right]+(\tau-\rho)\left[\left(x-x_{t_{n}}\right)^{T} X \dot{x}\right] \\
& -\left(x-x_{t_{n}}\right)^{T} X\left(x-x_{t_{n}}\right) .
\end{aligned}
$$

Since $\dot{V}=\dot{V}_{1}+\dot{V}_{2}+\dot{V}_{3}$, adding (25), (29), and (30) yields

$$
\begin{aligned}
\dot{V} \leq & \dot{x}^{T} P x+x^{T} P \dot{x}+\rho h_{i}^{T} R^{-1} h_{i} \\
& +\tau\left[\dot{x}-B\left(K_{j} x_{t_{n}}+k_{j}\right)\right]^{T} R\left[\dot{x}-B\left(K_{j} x_{t_{n}}+k_{j}\right)\right] \\
& -\left[x-x_{t_{n}}-\rho B\left(\left(K_{i} x_{t_{n}}+k_{i}\right)+w\right)\right]^{T} h_{i} \\
& -h_{i}^{T}\left[x-x_{t_{n}}-\rho B\left(\left(K_{i} x_{t_{n}}+k_{i}\right)+w\right)\right] \\
& +(\tau-\rho)\left[\dot{x}^{T} X\left(x-x_{t_{n}}\right)\right]+(\tau-\rho)\left[\left(x-x_{t_{n}}\right)^{T} X \dot{x}\right] \\
& -\left(x-x_{t_{n}}\right)^{T} X\left(x-x_{t_{n}}\right) .
\end{aligned}
$$

For $t \in\left(t_{n}, t_{n+1}\right)$ and $x(t) \in \mathcal{X}$ we consider the following three possibilities;

(1) $x(t) \notin \mathcal{R}^{*}$,

(2) $x(t) \in \mathcal{R}^{*}$ and $x_{t_{n}} \notin \mathcal{R}^{*}$,

(3) $x(t) \in \mathcal{R}^{*}$ and $x_{t_{n}} \in \mathcal{R}^{*}$.

The rest of the proof is divided into three parts corresponding to the above possibilities.

- Part 1: For $x(t) \in \mathcal{R}_{i} \neq \mathcal{R}^{*}$, based on (6), we have

$$
\dot{x}(t)=\left[\begin{array}{llll}
A_{i} & B K_{i} & B & a_{i}+B k_{i}
\end{array}\right] \zeta(t),
$$

and

$$
\dot{x}(t)-B\left(K_{j} x_{t_{n}}+k_{j}\right)=\left[\begin{array}{llll}
A_{i} & 0 & 0 & a_{i}
\end{array}\right] \zeta(t),
$$

with $\zeta(t)=\left[\begin{array}{llll}x^{T}(t) & x_{t_{n}}^{T} & w^{T}(t) & 1\end{array}\right]^{T} \in \mathbb{R}^{2 n_{x}+n_{u}+1}$. 
Replacing (32) and (33) in (31) and setting $h_{i}(t)=N_{i}^{T} \zeta(t)$ with $N_{i} \in \mathbb{R}^{\left(2 n_{x}+n_{u}+1\right) \times n_{x}}$, we can write

$$
\begin{aligned}
\dot{V} \leq \zeta^{T} & \left(\left[\begin{array}{llll}
A_{i} & B K_{i} & B & a_{i}+B k_{i}
\end{array}\right]^{T} P\left[\begin{array}{llll}
I & 0 & 0 & 0
\end{array}\right]\right. \\
& +\left[\begin{array}{llll}
I & 0 & 0 & 0
\end{array}\right]^{T} P\left[\begin{array}{llll}
A_{i} & B K_{i} & B & a_{i}+B k_{i}
\end{array}\right] \\
& +\rho N_{i} R^{-1} N_{i}^{T} \\
& +\tau\left[\begin{array}{llll}
A_{i} & 0 & 0 & a_{i}
\end{array}\right]^{T} R\left[\begin{array}{llll}
A_{i} & 0 & 0 & a_{i}
\end{array}\right] \\
& -\left[\begin{array}{lllll}
I & -I-\rho B K_{i} & -\rho B & -\rho B k_{i}
\end{array}\right]^{T} N_{i}^{T} \\
& -N_{i}\left[\begin{array}{lllll}
I & -I-\rho B K_{i} & -\rho B & -\rho B k_{i}
\end{array}\right] \\
& +(\tau-\rho)\left[\begin{array}{lllll}
A_{i} & B K_{i} & B & a_{i}+B k_{i}
\end{array}\right]^{T} X \\
& +\left(\begin{array}{lllll}
\tau-\rho) & -\left[\begin{array}{lllll}
I & -I & 0 & 0
\end{array}\right]^{T} X & 0
\end{array}\right] \\
& \left.-\left[\begin{array}{lllll}
I & -I & 0 & 0
\end{array}\right]^{T} X\left[\begin{array}{llll}
I & -I & 0 & 0
\end{array}\right]\right)
\end{aligned}
$$

Hence, for $\rho=0$, LMI (14) implies

$$
\dot{V}<-\eta x^{T} x-x_{t_{n}}^{T} x_{t_{n}}+\gamma w^{T} w-\eta-\zeta^{T} S_{1 i} \zeta .
$$

Using Schur complement, LMI (15) implies that (35) holds for $\rho=\tau$. Since (34) is affine in $\rho$, LMIs (14)-(15) are sufficient conditions for (35) to hold for any $\rho \in(0, \tau)$.

Recalling (7) and (9), we can write

$$
\|w\| \leq \Delta K\left\|x_{t_{n}}\right\|+\delta k .
$$

Considering (24) and (13), for $\left\|x_{t_{n}}\right\| \geq \mu_{\tau}$ we have

$$
\sqrt{\theta / \gamma}|| x_{t_{n}}\|-\Delta K\| x_{t_{n}} \| \geq \delta k
$$

Therefore, based on (36), for $\left\|x_{t_{n}}\right\| \geq \mu_{\tau}$ we can write

$$
\sqrt{\theta / \gamma}\left\|x_{t_{n}}\right\| \geq\|w\|
$$

Adding and subtracting $\theta x_{t_{n}}^{T} x_{t_{n}}, 0<\theta<1$, in inequality (35) and using (37), for $\left\|x_{t_{n}}\right\| \geq \mu_{\tau}$, we get

$$
\dot{V}<-\eta x^{T} x-(1-\theta) x_{t_{n}}^{T} x_{t_{n}}-\eta-\zeta^{T} S_{1 i} \zeta
$$

Furthermore, considering (34) for $\rho=0$, inequality (16) implies

$$
\dot{V}<\zeta^{T}\left(-\epsilon I-S_{1 i}-S_{3 i}\right) \zeta .
$$

Using Schur complement, inequality (17) implies that (39) holds at $\rho=\tau$. Since (34) is affine in $\rho$, inequalities (16)-(17) are sufficient conditions for (39) to hold for any $\rho \in(0, \tau)$.

According to Lemma $1, \zeta^{T} S_{1 i} \zeta>0$ if $x(t) \in \mathcal{R}_{i}$. Furthermore, using Lemma $2, \zeta^{T} S_{3 i} \zeta>0$ if $\left\|x_{t_{n}}\right\|<\mu_{\tau}$. Hence considering (38), LMIs (13)-(15) are sufficient conditions for $V$ to be strictly decreasing between two consecutive sampling times for $\left\|x_{t_{n}}\right\| \geq \mu_{\tau}$. Moreover, considering (39), inequalities (16)-(17) are sufficient conditions for $V$ to be strictly decreasing between two consecutive sampling times for $\left\|x_{t_{n}}\right\|<\mu_{\tau}$.

Therefore, inequalities (13)-(17) are sufficient conditions for $V$ to be strictly decreasing for any $t \in\left(t_{n}, t_{n+1}\right)$ and $x(t) \notin$ $\mathcal{R}^{*}$, regardless of the magnitude of $x_{t_{n}}$.

- Part 2: For $x(t) \in \mathcal{R}_{i}=\mathcal{R}^{*}$ and $x_{t_{n}} \notin \mathcal{R}^{*}$, based on Assumption 2, we have $a_{i}=0$ and $k_{i}=0$. Setting $a_{i}=0$ and $k_{i}=0$ in (34), for $\rho=0$, LMI (18) implies

$$
\dot{V}<-\eta x^{T} x-x_{t_{n}}^{T} x_{t_{n}}+\gamma w^{T} w-\eta+\zeta^{T}\left(-S_{1 i}+S_{2 i}\right) \zeta .
$$

Using Schur complement, LMI (19) implies that (40) holds for $\rho=\tau$. Since (34) is affine in $\rho$, LMIs (18)-(19) are sufficient conditions for (40) to hold for any $\rho \in(0, \tau)$.

Adding and subtracting $\theta x_{t_{n}}^{T} x_{t_{n}}$ with $0<\theta<1$ in (40) and recalling (37) for $\left\|x_{t_{n}}\right\| \geq \mu_{\tau}$, we get

$$
\dot{V}<-\eta x^{T} x-(1-\theta) x_{t_{n}}^{T} x_{t_{n}}-\eta+\zeta^{T}\left(-S_{1 i}+S_{2 i}\right) \zeta .
$$

Furthermore, considering (34) with $a_{i}=0, k_{i}=0$, and for $\rho=0$, inequality (20) implies

$$
\dot{V}<\zeta^{T}\left(-\epsilon I-S_{1 i}+S_{2 i}-S_{3 i}\right) \zeta .
$$

Using Schur complement, inequality (21) implies that (42) holds for $\rho=\tau$. Since (34) is affine in $\rho$, inequalities (20)(21) are sufficient conditions for (42) to hold for any $\rho \in(0, \tau)$.

Based on Lemma $1, \zeta^{T} S_{1 i} \zeta>0$ if $x(t) \in \mathcal{R}_{i}$. Furthermore, $\zeta^{T} S_{2 i} \zeta<0$ if $x_{t_{n}} \notin \mathcal{R}_{i}$. Finally, using Lemma 2, $\zeta^{T} S_{3 i} \zeta>0$ if $\left\|x_{t_{n}}\right\|<\mu_{\tau}$. Hence considering (41), LMIs (13) and (18)-(19) are sufficient conditions for $V$ to be strictly decreasing between two consecutive sampling times for $\left\|x_{t_{n}}\right\| \geq \mu_{\tau}$. Moreover, considering (42), inequalities (20)-(21) are sufficient conditions for $V$ to be strictly decreasing between two consecutive sampling times for $\left\|x_{t_{n}}\right\|<\mu_{\tau}$.

Therefore, inequalities (13) and (18)-(21) are sufficient conditions for $V$ to be strictly decreasing for any $t \in\left(t_{n}, t_{n+1}\right)$, $x(t) \in \mathcal{R}^{*}$, and $x_{t_{n}} \notin \mathcal{R}^{*}$, regardless of the magnitude of $x_{t_{n}}$

- Part 3: For $x(t) \in \mathcal{R}_{i}=\mathcal{R}^{*}$ and $x_{t_{n}} \in \mathcal{R}_{i}=\mathcal{R}^{*}$, According to (7) and Assumption 2, we have $a_{i}=0, k_{i}=0$, 
and $w=0$. Replacing $N_{i}$ by $\left[\begin{array}{ll}\widetilde{N}_{i}^{T} & 0_{n_{x} \times\left(n_{u}+1\right)}\end{array}\right]^{T}, \widetilde{N}_{i} \in$ $\mathbb{R}^{2 n_{x} \times n_{x}}$, and setting $a_{i}=0$ and $k_{i}=0$ in (34), LMI (22) implies

$$
\dot{V}<-\epsilon \widetilde{\zeta}^{T} \widetilde{\zeta}
$$

for $\rho=0$, where $\widetilde{\zeta}=\left[\begin{array}{ll}x^{T}(t) & x_{t_{n}}^{T}\end{array}\right]^{T}$. Using Schur complement, LMI (23) implies that (43) holds for $\rho=\tau$. Since (34) is affine in $\rho$, LMIs (22)-(23) are sufficient conditions for $V$ to be strictly decreasing for any $\rho \in(0, \tau), x(t) \in \mathcal{R}^{*}$, and $x_{t_{n}} \in \mathcal{R}^{*}$.

Therefore, inequalities (13)-(23) are sufficient conditions for $V$ to be strictly decreasing between any two consecutive sampling times over the state space. According to Assumption 3 , any sampling interval $\left(t_{n}, t_{n+1}\right), n \in \mathbb{N}$, has a length greater than or equal to $t_{\epsilon}>0$. Hence $\left.V\right|_{t_{n+1}^{-}}<\left.V\right|_{t_{n}}$, where $\left.V\right|_{t_{n+1}^{-}}=\lim _{t} \nearrow t_{n+1} V$.

Note that we computed $\dot{V}$ for the three possibilities in which the state vector $x(t)$ belongs in the state space $\mathcal{X}$. Therefore, we must ensure that $x(t)$ remains in $\mathcal{X}$ during the evolution of the sampled-data system. To this end, consider the following bounds on $V$ over the boundaries of the state space,

$$
\begin{aligned}
& C_{1}=\min _{c^{T} x=\sigma_{1}} V\left(x, x_{t}, \rho\right), \forall x_{t} \in \mathcal{C}, \rho \in[0, \tau), \\
& C_{M+1}=\min _{c^{T}} \min _{x=1} V\left(x, x_{t}, \rho\right), \forall x_{t} \in \mathcal{C}, \rho \in[0, \tau), \\
& C=\min \left\{C_{1}, C_{M+1}\right\}
\end{aligned}
$$

Note that the minima in (44) exist since $V_{1}$ is positive definite and radially unbounded, and $V_{2}$ and $V_{3}$ are non-negative. Let $\widetilde{C} \in(0, C)$ and define the set $\Omega$ as

$$
\Omega=\left\{\left(x, x_{t}, \rho\right) \mid V\left(x, x_{t}, \rho\right) \leq \widetilde{C}\right\} .
$$

Since $V$ is strictly decreasing in the sampling intervals and non-increasing at the sampling instants, the set $\Omega$ is forward invariant. Considering (44), it can be shown by contradiction that the projection of the set $\Omega$ onto $\mathcal{X}$ lies in the interior of $\mathcal{X}$. Therefore, for any trajectory starting in $\Omega$, the state vector remains in $\mathcal{X}$. Assuming that the system's trajectories start in $\Omega$, based on Lyapunov-Krasovskii theorem (Gu et al. (2003)), the closed-loop sampled data PWA slab system is uniformly asymptotically stable to the origin. Note that the Zeno phenomenon does not occur since, by Assumption 3, there exists $t_{\epsilon}>0$ such that $t_{n+1}-t_{n} \geq t_{\epsilon}$ for all $n \in \mathbb{N}$. $\square$

In the proof of Theorem 1, we showed that inequality (13) and inequalities (14)-(23) are sufficient conditions for the LKF to be decreasing, between two consecutive sampling times. Table 1 summarizes the correspondence between inequalities (13)-(23) and the portion of the state space that they refer to.
Table 1

The correspondence between inequalities of Theorem 1 and the state space.

\begin{tabular}{|c|c|c|}
\cline { 2 - 3 } \multicolumn{1}{c|}{} & $\left\|x_{t_{n}}\right\| \geq \mu_{\tau}$ & $\left\|x_{t_{n}}\right\|<\mu_{\tau}$ \\
\hline$x(t) \notin \mathcal{R}^{*}$ & (13) and (14)-(15) & (16)-(17) \\
\hline$x(t) \in \mathcal{R}^{*}$ and $x_{t_{n}} \notin \mathcal{R}^{*}$ & (13) and (18)-(19) & (20)-(21) \\
\hline$x(t) \in \mathcal{R}^{*}$ and $x_{t_{n}} \in \mathcal{R}^{*}$ & \multicolumn{2}{|c|}{ (22)-(23) } \\
\hline
\end{tabular}

Remark 1 In intuitive terms, relaxing Assumption 3 by letting the sampling intervals approach zero, yields $\tau \rightarrow 0$ and $x(t)=x_{t_{n}}$ for $t_{n} \leq t<t_{n+1}$. Therefore, $V_{2}$ and $V_{3}$ vanish and the inequalities in Theorem 1 reduce to the LMI conditions for stability of continuous-time PWA slab systems; Rodrigues and Boyd (2005).

We now present the result for a PWA slab system in feedback with a sampled-data PWL controller as a corollary.

Corollary 1 Consider the sampled-data PWA slab system defined in (6) and (7) subject to Assumptions 1-3. Assume that the controller is piecewise linear $(P W L)$, i.e. $k_{i}=$ $0, \forall i \in \mathcal{I}$. The system is uniformly asymptotically stable to the origin if there exist symmetric positive definite matrices $P, R$, and $X$, matrices $\widetilde{N}$ and $N_{i}$, with appropriate dimensions, and positive scalars $\gamma, \theta<1, \eta, \lambda_{i}, \sigma$, and $\epsilon$, with $i \in \mathcal{I}$, satisfying (13)-(15), (18)-(19), and (22)-(23).

PROOF. Since $k_{i}=0$ for all $i \in \mathcal{I}$, we get $\delta k=0$. Hence, equation (24) yields $\mu_{\tau}=0$. According to the proof of Theorem 1, LMIs (13)-(15), (18)-(19), and (22)-(23) are sufficient conditions for the LKF (12) to be strictly decreasing for any $t \in\left(t_{n}, t_{n+1}\right)$ and $\left\|x_{t_{n}}\right\| \geq 0$ (i.e. the whole state space). Since the LKF is non-increasing at the sampling instants, similar to the proof of Theorem 1, a forward invariant set can be found. Assuming that the trajectories start in the invariant set, the closed-loop sampled data PWA slab system is uniformly asymptotically stable to the origin.

Remark 2 For PWL controllers we have $\mu_{\tau}=0$. Therefore according to Table 1, Corollary 1 contains only those inequalities of Theorem 1 that correspond to $\left\|x_{t_{n}}\right\| \geq \mu_{\tau}$. Consequently, the inequalities in Corollary 1 can be solved efficiently as a set of LMIs. This case was studied in details in Moarref and Rodrigues (2011). For PWA controllers, however, the inequalities in Theorem 1 do not constitute a set of LMIs.

Note that the matrix $S_{3 i}$ is a nonlinear function of the variables $\gamma$ and $\theta$. Hence, inequalities (13)-(23) cannot be solved simultaneously using LMI solvers. However, inequalities (13)-(15), (18)-(19), and (22)-(23) are linear in $\gamma$ and $\theta$ and constitute a set of LMIs. Moreover, treating $\gamma$ and $\theta$ as constant parameters, inequalities (16)-(17) and (20)-(21) become a set of LMIs. Based on the above observations, we propose a two-phase algorithm for solving inequalities (13)(23). To this end, consider the following remark. 
Remark 3 The variable $0<\theta<1$ appears only in inequality (13) and the matrix $S_{3 i}$. Without loss of generality, we assume $\theta=1$-eps, where eps is the machine epsilon. To justify this assumption, note that if (13) is satisfied for a $\theta$, it is also satisfied for any larger $\theta$. Moreover, based on (24), a larger $\theta$ yields a smaller $\mu_{\tau}$, which in turn provides a tighter bound on the mismatch vector $w$ (see Lemma 2). A tighter bound on $w$ makes LMIs (16)-(17) and (20)-(21) less conservative through the $\mathcal{S}$-procedure term $S_{3 i}$. This in turn allows the algorithm to yield a larger lower bound on the longest sampling interval that preserves asymptotic stability.

Algorithm 1 finds a lower bound on the longest interval between two consecutive sampling times $\tau_{\max }$ which preserves asymptotic stability. In the first phase of the algorithm, given $\tau$, we solve the following optimization problem.

\section{Problem 1}

$$
\begin{aligned}
\text { minimize } & \gamma \\
\text { subject to } & P>0, R>0, X>0, \gamma>0 \\
& \eta>0, \sigma>0, \lambda_{i}>0, i \in \mathcal{I} \\
& (13)-(15),(18)-(19), \text { and }(22)-(23) .
\end{aligned}
$$

If Problem 1 is feasible, according to Table 1 , the LKF is decreasing for any $t \in\left(t_{n}, t_{n+1}\right)$ and $\left\|x_{t_{n}}\right\| \geq \mu_{\tau}$. Note that minimizing $\gamma$ leads to a smaller $\mu_{\tau}$ which relaxes the inequalities that will be solved in the next phase (see Remark 3). Treating $\gamma, P, R$, and $X$ as constant parameters computed in Problem 1, we solve the following feasibility problem in the second phase.

\section{Problem 2}

$$
\begin{array}{ll}
\text { find } & \epsilon>0, \sigma>0, \lambda_{i}>0, \Lambda_{i} \succeq 0, i \in \mathcal{I} \\
\text { subject to } & (16)-(17) \text { and }(20)-(21) .
\end{array}
$$

If Problem 2 is feasible, based on Table 1, the LKF is decreasing for any $t \in\left(t_{n}, t_{n+1}\right)$ and $\left\|x_{t_{n}}\right\|<\mu_{\tau}$.

Remark 4 In Problem 2, matrices $P, R$, and $X$ are treated as constant parameters and replaced with the numerical values computed in Problem 1, so that the same LKF is used both outside and inside the ball of radius $\mu_{\tau}$.

In the next section, we use Algorithm 1 to compute $\tau_{\max }$ in a unicycle example.

\section{Numerical Example}

Consider the line following example of Rodrigues and Boyd (2005), whose objective is to control a unicycle to follow the line $y=0$ in the $x-y$ plane (see Fig. 1). The dynamics

\begin{tabular}{|l|l|}
\hline Name & Algorithm 1 \\
\hline Goal & $\begin{array}{l}\text { Find a lower bound on the longest interval be- } \\
\text { tween two consecutive sampling times }\left(\tau_{\max }\right) \\
\text { that preserves asymptotic stability }\end{array}$ \\
\hline Inputs & $\begin{array}{l}\text { A PWA slab system and a PWA slab } \\
\text { continuous-time controller }\end{array}$ \\
\hline Outputs & $\begin{array}{l}\text { A lower bound on the longest interval between } \\
\text { two consecutive sampling times }\left(\tau_{\max }\right) \text { and an } \\
\text { LKF which proves asymptotic stability }\end{array}$ \\
\hline
\end{tabular}

Initialization: set $\tau_{\max }:=0, \theta:=1-\mathbf{e p s}, \tau_{l}:=0, \tau_{u}:=\mathcal{M}$, where $\mathcal{M}$ is a large number, and choose a finite threshold $>0$

while $\tau_{u}-\tau_{l}>$ threshold:

set $\tau:=\left(\tau_{l}+\tau_{u}\right) / 2$

if Problem 1 is infeasible:

set $\tau_{u}:=\tau$

elseif the controller is PWL:

$$
\text { set } \tau_{\max }:=\tau \text { and } \tau_{l}:=\tau
$$

else:

(Using $\gamma, P, R$, and $X$ from solution of Problem 1)

if Problem 2 is infeasible:

$$
\text { set } \tau_{u}:=\tau
$$

else:

$$
\text { set } \tau_{\max }:=\tau \text { and } \tau_{l}:=\tau
$$

of the system are represented by

$$
\left[\begin{array}{c}
\dot{\psi} \\
\dot{r} \\
\dot{y}
\end{array}\right]=\left[\begin{array}{ccc}
0 & 1 & 0 \\
0 & -k / I & 0 \\
0 & 0 & 0
\end{array}\right]\left[\begin{array}{l}
\psi \\
r \\
y
\end{array}\right]+\left[\begin{array}{c}
0 \\
0 \\
v \sin (\psi)
\end{array}\right]+\left[\begin{array}{c}
0 \\
1 / I \\
0
\end{array}\right] u,
$$

where $\psi$ and $r$ are the heading angle and its time derivative, respectively, $y$ is the distance from the line $y=0, v$ represents the unicycle's velocity, $u$ is the torque input about the $z$ axis, $I=1\left(\mathrm{kgm}^{2}\right)$ is the unicycle's moment of inertia with respect to its center of mass, and $k=0.01$ (Nms) is the damping coefficient. The state vector of the system is represented by $z=\left[\begin{array}{lll}\psi & r & y\end{array}\right]^{T}$. We assume that the unicycle has a constant velocity $v=1(\mathrm{~m} / \mathrm{s})$ and the heading angle $\psi$ is restricted to the interval $[-3 \pi / 5,3 \pi / 5]$, i.e. the state space is defined as $\mathcal{Z}=[-3 \pi / 5,3 \pi / 5] \times \mathbb{R}^{2}$.

The system's nonlinearity, $\sin (\psi)$, is approximated by a PWA function. The PWA approximation is defined over the following five regions:

$$
\begin{aligned}
& \mathcal{R}_{1}=\left\{z \in \mathbb{R}^{3} \mid \psi \in(-3 \pi / 5,-\pi / 5)\right\}, \\
& \mathcal{R}_{2}=\left\{z \in \mathbb{R}^{3} \mid \psi \in(-\pi / 5,-\pi / 15)\right\}, \\
& \mathcal{R}_{3}=\left\{z \in \mathbb{R}^{3} \mid \psi \in(-\pi / 15, \pi / 15)\right\}, \\
& \mathcal{R}_{4}=\left\{z \in \mathbb{R}^{3} \mid \psi \in(\pi / 15, \pi / 5)\right\},
\end{aligned}
$$




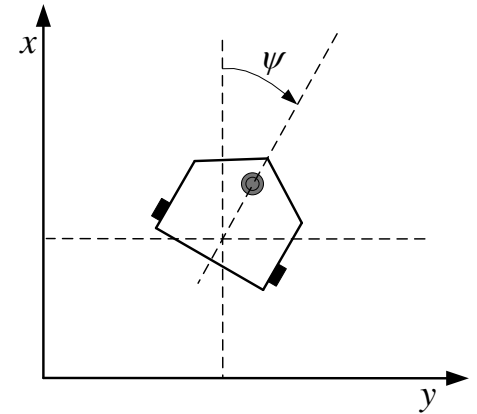

Fig. 1. Unicycle path following example

$\mathcal{R}_{5}=\left\{z \in \mathbb{R}^{3} \mid \psi \in(\pi / 5,3 \pi / 5)\right\}$.

Consider the PWA controller

$$
u=K_{i} z+k_{i}, \text { for } z \in \mathcal{R}_{i}, i \in\{1, \ldots, 5\},
$$

with

$$
\begin{array}{ll}
K_{1}=[-49.907-9.468-13.925], & k_{1}=-0.617, \\
K_{2}=[-48.315-9.330-13.812], & k_{2}=0.384, \\
K_{3}=[-50.147-9.468-13.742], & k_{3}=0, \\
K_{4}=[-48.316-9.330-13.812], & k_{4}=-0.384, \\
K_{5}=[-49.907-9.468-13.925], & k_{5}=0.617 .
\end{array}
$$

The vector gains $K_{i}, i \in\{1, \ldots, 5\}$, are taken from the PWL controller proposed in Rodrigues and Boyd (2005). The affine gains $k_{i}, i \in\{1, \ldots, 5\}$, are added to the controller such that the continuous-time PWA controller becomes continuous at the boundaries of the regions. Our goal is to find a lower bound on the longest interval between two consecutive sampling times such that asymptotic stability is guaranteed. Using Algorithm 1, with $\tau_{u}=0.2$ and threshold=0.001, after eight iterations, we get

$$
\tau_{\max }=0.104(\mathrm{sec})
$$

and

$$
\begin{aligned}
P & =\left[\begin{array}{ccc}
14.75 & 0.45 & 4.20 \\
0.45 & 0.19 & 0.13 \\
4.20 & 0.13 & 5.80
\end{array}\right], X=\left[\begin{array}{ccc}
98.74 & 9.75 & 30.12 \\
9.75 & 1.37 & 6.73 \\
30.12 & 6.73 & 790.61
\end{array}\right] \\
R & =\left[\begin{array}{ccc}
8.29 & 72.57 & 1.18 \\
72.57 & 7112.51 & -17.32 \\
1.18 & -17.32 & 5.00
\end{array}\right]
\end{aligned}
$$

Similar to (45), an invariant set $\Omega^{\prime}$ can be computed by considering the quadratic term $V_{1}$ in the LKF, i.e.

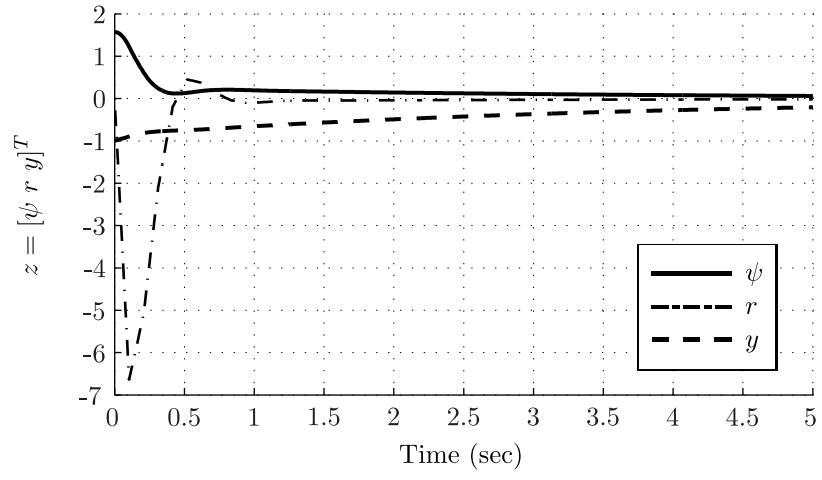

Fig. 2. Unicycle's states for $T_{s}=\tau_{\max }$.

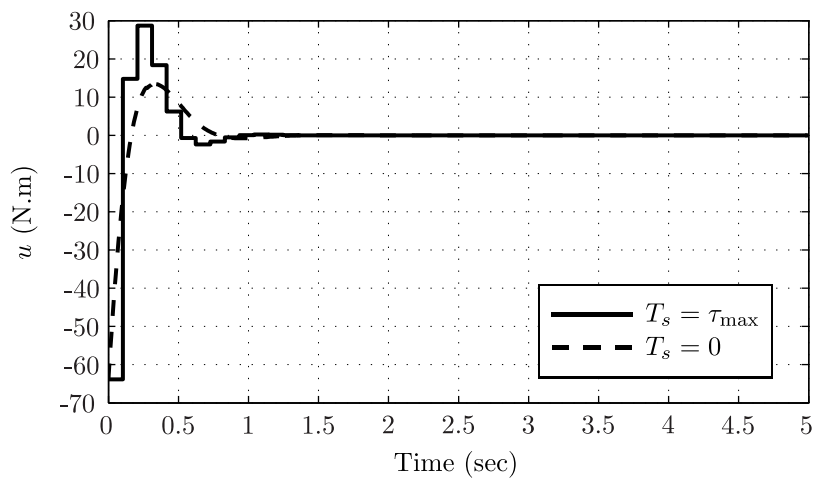

Fig. 3. Control input for $T_{s}=\tau_{\max }$ and $T_{s}=0$.

$\Omega^{\prime}=\left\{\left(z, z_{t}, \rho\right) \mid V\left(z, z_{t}, \rho\right) \leq \widetilde{C^{\prime}}\right\}$, where $\widetilde{C^{\prime}} \in\left(0, C^{\prime}\right)$ and $C^{\prime}=\min _{|\psi|=3 \pi / 5} V_{1}\left(z, z_{t}, \rho\right) \leq \min _{|\psi|=3 \pi / 5} V\left(z, z_{t}, \rho\right)$. Since $V_{1}=z^{T} P z$, with $P$ computed in (48), we find $C^{\prime}=39.245$. Let $\widetilde{C^{\prime}}=39.24<C^{\prime}$ and choose the system's trajectories to start in $\Omega^{\prime}$. Theorem 1 guarantees that if controller (47) is implemented in the unicycle via sample-and-hold, with variable sampling rates greater than $1 / \tau_{\max }=9.62(\mathrm{~Hz})$, the PWA closed-loop system asymptotically converges to the origin.

Figures 2- 3, illustrate the simulation results for the unicycle system (46) with PWA feedback (47). The initial condition is $z_{0}(\alpha)=[\pi / 2,0,-1]^{T},-0.104 \leq \alpha \leq 0$, and $\rho(0)=0$. The simulation is performed for sampling time $T_{s}=\tau_{\max }=0.104(\mathrm{sec})$. According to Fig. 2 the state vector asymptotically converges to the origin. The solid line in Fig. 3 shows the torque input for the sampled-data PWA controller. The dashed curve in Fig. 3 illustrates the torque input if the PWA controller was implemented in continuous-time. As expected, more control energy is required to stabilize the system with the sample-and-hold controller.

Simulating the system with the same initial condition $z_{0}$ for $T_{s}=0.213$ (sec), the closed-loop sampled-data trajectories do not converge to the origin. Therefore, in this example, the error in the computed lower bound on the longest sampling interval that preserves asymptotic stability is at most $51 \%$. 
Table 2

Comparison of two stability theorems applied to the unicycle problem

\begin{tabular}{|c|c|c|}
\hline Method & Stability Result & $\tau_{\max }(\mathrm{sec})$ \\
\hline Theorem 1 in Samadi and Rodrigues (2009b) & Convergence to the invariant set $\left\{V \leq 4.296 \times 10^{6}\right\}$ & 0.098 \\
\hline Algorithm 1 in this paper & Asymptotic stability to the origin & 0.104 \\
\hline
\end{tabular}

Still, as shown in Table 2, the $\tau_{\max }$ provided by Algorithm 1 is less conservative than the previous results in the literature. Moreover, Algorithm 1 provides a stronger stability result (asymptotic stability to the origin) than Theorem 1 in Samadi and Rodrigues (2009b).

\section{Conclusion}

In this paper we presented a less conservative LKF for studying PWA systems with time delay. Based on the new LKF, sufficient conditions were provided for asymptotic stability of sampled-data PWA slab systems to the origin. It was shown that these conditions become LMIs in the case of a PWL controller. Finally, we presented an algorithm for finding a lower bound on the maximum delay that preserves asymptotic stability. The output of the algorithm provides an upper bound on the minimum sampling frequency that guarantees asymptotic stability of the sampled data system. It was shown that our results compare favorably with the results available in the literature.

\section{References}

Azuma, S., Imura, J., 2003. Optimal control of sampleddata piecewise affine systems and its application to CPU processing control. In: Proc. 42nd IEEE Conference on Decision and Control. pp. 161-166.

Chen, T., Francis, B., 1995. Optimal Sampled-Data Control Systems. Springer-Verlag.

Gu, K., Kharitonov, V. L., Chen, J., 2003. Stability of TimeDelay Systems. Birkhauser.

Hale, J. K., Lunel, S. M. V., 1993. Introduction to functional differential equations. Springer.

Hassibi, A., Boyd, S., 1998. Quadratic stabilization and control of piecewise-linear systems. In: Proc. American Control Conference. pp. 3659-3664.

Imura, J., 2003. Optimal continuous-time control of sampled-data piecewise affine systems. In: Proc. American Control Conference. pp. 5317-5322.

Johansson, M., 2002. Piecewise Linear Control SystemsA Computational Approach. Vol. 284. Lecture notes in control and information sciences, Springer-Verlag.

Johansson, M., Rantzer, A., 1998. Computation of piecewise quadratic Lyapunov functions for hybrid systems. IEEE Transactions on Automatic Control 43 (4), 555-559.

Laila, D. S., Nešić, D., Teel, A. R., 2002. Open and closed loop dissipation inequalities under sampling and controller emulation. European Journal of Control 8 (2), 109125.

Moarref, M., Rodrigues, L., 2011. Asymptotic stability of piecewise affine systems with sampled-data piecewise lin- ear controllers. In: Proc. 50th IEEE Conference on Decision and Control and European Control Conference. pp. 8315-8320.

Naghshtabrizi, P., Hespanha, J. P., Teel, A. R., 2008. Exponential stability of impulsive systems with application to uncertain sampled-data systems. Systems \& Control Letters 57 (5), 378-385.

Nešić, D., Teel, A. R., 2004. A framework for stabilization of nonlinear sampled-data systems based on their approximate discrete-time models. IEEE Transactions on Automatic Control 49 (7), 1103-1122.

Nešić, D., Teel, A. R., Kokotovic, P., Dec. 1999. Sufficient conditions for stabilization of sampled-data nonlinear systems via discrete-time approximations. Systems \& Control Letters 38 (4-5), 259-270.

Rodrigues, L., 2004. Stability analysis of piecewise-affine systems using controlled invariant sets. Systems \& Control Letters 53 (2), 157-169.

Rodrigues, L., 2007. Stability of sampled-data piecewiseaffine systems under state feedback. Automatica 43 (7), 1249-1256.

Rodrigues, L., Boyd, S., 2005. Piecewise-affine state feedback for piecewise-affine slab systems using convex optimization. Systems \& Control Letters 54 (9), 835-853.

Rodrigues, L., How, J., 2001. Automated control design for a piecewise-affine approximation of a class of nonlinear systems. In: Proc. American Control Conference. pp. 3189-3194.

Samadi, B., Rodrigues, L., 2009a. A duality-based convex optimization approach to $L_{2}$-gain control of piecewise affine slab differential inclusions. Automatica 45 (3), 812816.

Samadi, B., Rodrigues, L., 2009b. Stability of sampled-data piecewise affine systems: A time-delay approach. Automatica 45 (9), 1995-2001.

Teel, A. R., Nešić, D., Kokotovic, P., 1998. A note on inputto-state stability of sampled-data nonlinear systems. In: Proc. 37th IEEE Conference on Decision and Control. pp. 2473-2478. 